PROCEEDINGS OF THE

AMERICAN MATHEMATICAL SOCIETY

Volume 129, Number 8 , Pages 2209-2217

S 0002-9939(00)05863-9

Article electronically published on December 28, 2000

\title{
SPLITTING SETS IN INTEGRAL DOMAINS
}

\author{
D. D. ANDERSON AND MUHAMMAD ZAFRULLAH
}

(Communicated by Wolmer V. Vasconcelos)

\begin{abstract}
Let $D$ be an integral domain. A saturated multiplicatively closed subset $S$ of $D$ is a splitting set if each nonzero $d \in D$ may be written as $d=s a$ where $s \in S$ and $s^{\prime} D \cap a D=s^{\prime} a D$ for all $s^{\prime} \in S$. We show that if $S$ is a splitting set in $D$, then $S U\left(D_{N}\right)$ is a splitting set in $D_{N}, N$ a multiplicatively closed subset of $D$, and that $S \subseteq D$ is a splitting set in $D[X] \Longleftrightarrow S$ is an lcm splitting set of $D$, i.e., $S$ is a splitting set of $D$ with the further property that $s D \cap d D$ is principal for all $s \in S$ and $d \in D$. Several new characterizations and applications of splitting sets are given.
\end{abstract}

\section{INTRODUCTION}

Throughout this paper $D$ denotes an integral domain with quotient field $K$. As usual, $D^{*}=D-\{0\}$ and $U(D)$ is the group of units of $D$.

A saturated multiplicatively closed subset $S$ of $D^{*}$ is said to be a splitting set if for each $d \in D^{*}$ we can write $d=s a$ for some $s \in S$ and $a \in D$ with $s^{\prime} D \cap a D=s^{\prime} a D$ for all $s^{\prime} \in S$. A splitting set is said to be an lcm splitting set if for each $s \in S$ and $d \in D, s D \cap d D$ is principal.

Perhaps the most important example of an lcm splitting set is as follows. A set $\left\{p_{\alpha}\right\}$ of nonzero principal primes is a splitting set of principal primes if (a) for each $\alpha, \bigcap_{n=1}^{\infty} p_{\alpha}^{n} D=0$ (or equivalently, ht $p_{\alpha} D=1$ ) and (b) for any sequence $\left\{p_{\alpha_{n}}\right\}$ of nonassociate members of $\left\{p_{\alpha}\right\}, \bigcap_{n=1}^{\infty} p_{\alpha_{n}} D=0$. Then $S=\left\{u p_{\alpha_{1}} \cdots p_{\alpha_{n}} \mid u \in\right.$ $\left.U(D), p_{\alpha_{i}} \in\left\{p_{\alpha}\right\}, n \geq 0\right\}$ is an lcm splitting set. In fact, if $D$ is an atomic integral domain (i.e., every nonzero nonunit of $D$ is a product of irreducible elements), then a multiplicatively closed subset $S$ of $D$ is an lcm splitting set if and only if $S$ is generated by a splitting set of principal primes ([1 Corollary 2.7]). Several characterizations of multiplicatively closed subsets generated by a set of splitting primes may be found in [1. Proposition 2.6]. Splitting sets of primes were probably first used by Nagata [11 to obtain his useful result that if $D$ is Noetherian and $S$ is a multiplicatively closed subset of $D$ generated by principal primes with $D_{S}$ a UFD, then $D$ is a UFD.

Splitting sets were first considered by Mott and Schexnayder [10. They gave a condition ([10. Proposition 4.1]), essentially (44) of Theorem [1.2, for a multiplicatively closed subset $S$ of $D$ to have $\langle S\rangle$, the subgroup $\left\{s_{1} s_{2}^{-1} D \mid s_{1}, s_{2} \in S\right\}$ of the group $P(D)$ of nonzero principal fractional ideals of $D$, to be a cardinal summand of $P(D)$. They showed that if $S$ is generated by a splitting set of principal primes,

Received by the editors May 5, 1999 and, in revised form, December 18, 1999.

1991 Mathematics Subject Classification. Primary 13A05, 13A15, 13 G05.

Key words and phrases. Splitting sets. 
then $\langle S\rangle$ is a cardinal summand of $P(D)$. They then observed that this approach could be used to prove known results such as if $D$ is a GCD domain (respectively, $\mathrm{UFD}$ ), then $D[X]$ is a GCD domain (respectively, UFD); and certain "Nagatatype" theorems: if $S$ is generated by a splitting set of principal primes and if $D_{S}$ is a GCD domain (respectively, UFD), then $D$ is a GCD domain (respectively, UFD). Other Nagata-type theorems using splitting sets of primes were given by Gabelli and Roitman [7] and Roitman [12]. Additional Nagata-type theorems were given in 2. where an alternative characterization for $\langle S\rangle$ being a cardinal summand was used, namely $S$ being a splitting set. Splitting sets and lcm splitting sets were then considered in detail in [1. Splitting sets have also been used by Mott [9] to compute the group of divisibility of a Rees ring and by D.F. Anderson et al. [5] to study elasticity of factorizations. Also, lcm splitting sets (not generated by principal primes) were first used by Gilmer and Parker [8] in their study of divisibility properties in semigroup rings.

As previously mentioned, the notion of a splitting set has been useful in studying factorizations and explaining why statements like Nagata's theorem on UFD's are possible in various situations. Our aim here is to bring together the various characterizations and applications of splitting sets and their variations and add a few more to the list.

In Section II we show that if $S$ is an (lcm) splitting set of $D$, then $S U\left(D_{N}\right)$ is an $(\mathrm{lcm})$ splitting set of $D_{N}$ where $N$ is a multiplicatively closed subset of $D$ and that $S \subseteq D^{*}$ is a splitting set of $D[X]$ if and only if $S$ is an lcm splitting set of $D$. Then several new characterizations and applications of splitting sets are given.

We say that $x, y \in D^{*}$ are $v$-coprime if $(x, y)_{v}=D$, or equivalently, $x D \cap y D=$ $x y D$. Here as usual for a nonzero fractional ideal $I$ of $D, I_{v}=\left(I^{-1}\right)^{-1}$. Let $X$ be a nonempty subset of $D^{*}$. We say that $t \in D^{*}$ is $v$-coprime to $X$ if $t$ is $v$-coprime to each $x \in X$. Then the set $T_{X}=\left\{t \in D^{*} \mid t\right.$ is $v$-coprime to $\left.X\right\}$ is easily seen to be a saturated multiplicatively closed subset of $D$. We refer to $T_{X}$ as the $m$-complement of $X$ and may drop the subscript if no confusion is possible. The following result relates $v$-coprimeness to overrings. As usual, for $s \in D^{*}, D_{s}=D_{S}$ where $S=\left\{s^{n} \mid n \geq 0\right\}$.

Proposition 1.1. Let $S$ and $T$ be multiplicatively closed subsets of $D$. Then $S$ and $T$ are $v$-coprime if and only if $D=D_{S} \cap D_{T}$ and $s, t \in D^{*}$ are $v$-coprime if and only if $D=D_{s} \cap D_{t}$.

Proof. $(\Rightarrow)$ Let $0 \neq x \in D_{S} \cap D_{T}$. So there exist $s \in S$ and $t \in T$ with $s x, t x \in D$. Hence $D \supseteq(s x, t x)_{v}=x(s, t)_{v}=x D$, and hence $D=D_{S} \cap D_{T}$.

$(\Leftarrow)$ Suppose $D=D_{S} \cap D_{T}$. Let $s \in S$ and $t \in T$. Then $s D \cap t D=s\left(D_{S} \cap D_{T}\right) \cap$ $t\left(D_{S} \cap D_{T}\right)=s D_{S} \cap s D_{T} \cap t D_{S} \cap t D_{T}=s D_{S} \cap s t D_{T} \cap s t D_{S} \cap t D_{T}=s t D_{S} \cap s t D_{T}=$ st $\left(D_{S} \cap D_{T}\right)=s t D$.

A multiplicatively closed subset $S$ of $D$ with $U(D) \subseteq S$ is a splitting set if and only if for $T=T_{S}$, we have $S T=\{s t \mid s \in S, t \in T\}=D^{*}$. Note that if $S$ is a splitting set with $m$-complement $T$, then $T$ is also a splitting set with $S$ for its $m$-complement. The following two theorems from [1] give several characterizations of splitting sets and lcm splitting sets.

Theorem 1.2 ([1, Theorem 2.2]). The following conditions are equivalent for a saturated multiplicatively closed subset of an integral domain $D$.

(1) $S$ is a splitting set. 
(2) $\langle S\rangle$ is a cardinal summand of $P(D)$, that is, there is a subgroup $H$ of $P(D)$ with $\langle S\rangle \oplus_{c} H=P(D)$.

(3) If $A$ is an integral principal ideal of $D_{S}$, then $A \cap D$ is a principal ideal of $D$.

(4) There exists a multiplicatively closed subset $T$ of $D$ such that:

(a) each element $d \in D^{*}$ may be written as $d=$ st where $s \in S$ and $t \in T$, and

(b) one of the following equivalent four conditions holds:

(i) If $s t=s^{\prime} t^{\prime}$, where $s, s^{\prime} \in S$ and $t, t^{\prime} \in T$, then $s^{\prime}=s u$ and $t^{\prime}=t u^{-1}$ where $u \in U(D)$.

(ii) If $d=$ st where $s \in S$ and $t \in T$, then $d D_{S} \cap D=t D$.

(iii) For each $s \in S$ and $t \in T, s D \cap t D=s t D$.

(iv) For each $t \in T, t D_{S} \cap D=t D$.

Theorem 1.3 ([1, Proposition 2.4]). The following conditions are equivalent for a splitting multiplicatively closed subset $S$ of $D$.

(1) $S$ is lcm splitting.

(2) For $s_{1}, s_{2} \in S, s_{1} D \cap s_{2} D$ is principal.

(3) For $s_{1}, s_{2} \in S, s_{1} D \cap s_{2} D=s D$ for some $s \in S$.

(4) $D_{T}$ is a GCD domain where $T$ is the $m$-complement for $S$.

We follow the notation and terminology of [1]. For a nonzero fractional ideal $I$ of $D, I_{t}=\bigcup\left\{\left(a_{1}, \ldots, a_{n}\right)_{v} \mid a_{1}, \ldots, a_{n} \in I-\{0\}\right\}$ and $I$ is a $t$-ideal if $I=I_{t}$. The set of maximal $t$-ideals of $D$ is denoted by $t$ - $\max (D)$. For $f=a_{0}+a_{1} X+\cdots+a_{n} X^{n} \in$ $D[X], A_{f}=\left(a_{0}, \ldots, a_{n}\right)$, the content of $f$.

\section{Splitting Sets}

We begin this section by proving two stability theorems for splitting sets. Theorem 2.1 gives that if $S$ is a splitting set of the integral domain $D$ and $N$ is a multiplicatively closed subset of $D$, then $S U\left(D_{N}\right)$ is a splitting set in $D_{N}$. Theorem 2.2 shows that a multiplicatively closed subset $S$ of $D$ is a splitting set for $D[X]$ if and only if $S$ is an lcm splitting set in $D$.

Theorem 2.1. Let $D$ be an integral domain and $S$ a splitting set in $D$ with $m$ complement $T$. Let $N$ be a multiplicatively closed subset of $D$. Then $\bar{S}=S U\left(D_{N}\right)$ is a splitting set in $D_{N}$ with m-complement $\bar{T}=T U\left(D_{N}\right)$. Moreover, if $S$ is an lcm splitting set in $D$, then $\bar{S}$ is an lcm splitting set in $D_{N}$.

Proof. Now $\bar{S}$ and $\bar{T}$ are multiplicatively closed subsets of $D_{N}$ containing $U\left(D_{N}\right)$. Clearly $\bar{S} \bar{T}=D_{N}^{*}$. For $s u_{1} \in S$ and $t u_{2} \in \bar{T}$ where $s \in S, t \in T$ and $u_{1}, u_{2} \in$ $U\left(D_{N}\right), s u_{1} D_{N} \cap t u_{2} D_{N}=s D_{N} \cap t D_{N}=(s D \cap t D)_{N}=s t D_{N}=\left(s u_{1}\right)\left(t u_{2}\right) D_{N}$. So $\bar{T}$ is contained in the $m$-complement of $\bar{S}$. By the paragraph after Proposition 1.1, $\bar{S}$ is a splitting set with $m$-complement $\bar{T}$.

Suppose that $S$ is an lcm splitting set in $D$. Then $D_{T}$ is a GCD domain by Theorem 1.3. Hence $\left(D_{N}\right)_{\bar{T}}$ being a localization of $D_{T}$ is a GCD domain. Again by Theorem $1.3, \bar{S}$ is an lcm splitting set in $D_{N}$.

Theorem 2.2. Let $S$ be a multiplicatively closed subset of the integral domain $D$. If $S$ is a splitting set in $D[X]$, then $S$ is an lcm splitting set in $D$ and in $D[X]$. Conversely, if $S$ is an lcm splitting set of $D$ with $m$-complement $T$, then $S$ is an lcm splitting set of $D[X]$ with m-complement $T^{\prime}=\left\{f \in D[X] \mid\left(A_{f} D_{T}\right)_{t}=D_{T}\right\}$. 
Proof. First, suppose $S$ is a splitting set in $D[X]$. Let $T^{\prime}$ be the $m$-complement of $S$ in $D[X]$. Clearly $S$ is a splitting set in $D$. Let $s_{0}, s_{1} \in S$. For some $s \in S$ and $f \in T^{\prime}$ we have $s_{0}+s_{1} X=s f$, say $f=t_{0}+t_{1} X$. Let $a \in s_{0} D \cap s_{1} D$, thus $a=a_{0} s_{0}=a_{1} s_{1}$ with $a_{0}, a_{1} \in D$. Since $f \in T^{\prime}$ and since $f=t_{0}+t_{1} X$ divides $s_{0} s_{1}\left(a_{1}+a_{0} X\right)$ in $D[X]$, we see that $t_{0}+t_{1} X \mid a_{1}+a_{0} X$ in $D[X]$. Hence $t_{0} \mid a_{1}$ in $D$, which implies that $a \in u D$, where $u=t_{0} s_{1}=t_{1} s_{0}=s t_{0} t_{1}$. We obtain that $s_{0} D \cap s_{1} D=u D$, hence $s_{0} D[X] \cap s_{1} D[X]=u D[X]$. By Theorem 1.3 we conclude that $S$ is an lcm splitting set in $D$ and in $D[X]$.

Suppose that $S$ is an lcm splitting set of $D$ with $m$-complement $T$. Now $S$ is a saturated multiplicatively closed subset of $D[X]$. Since $D=D_{S} \cap D_{T}, D[X]=$ $D_{S}[X] \cap D_{T}[X]$. Let $0 \neq f=a_{0}+a_{1} X+\cdots+a_{n} X^{n} \in D[X]$. For each nonzero $a_{i}, a_{i}=s_{i} t_{i}$ where $s_{i} \in S$ and $t_{i} \in T$. Now since $S$ is an $1 \mathrm{~cm}$ splitting set, $s=\operatorname{GCD}\left(\left\{s_{i} \mid s_{i} \neq 0\right\}\right)$ exists and $s D=\left(\left\{s_{i} \mid s_{i} \neq 0\right\}\right)_{t}$. Let $f=s f^{\prime}$ where $f^{\prime}=$ $\left(\frac{s_{0}}{s}\right) t_{0}+\left(\frac{s_{1}}{s}\right) t_{1} X+\cdots+\left(\frac{s_{n}}{s}\right) t_{n} X^{n}$. Note that $\left(A_{f^{\prime}}\right)_{t}=\left(\left(\frac{s_{0}}{s}\right) t_{0}, \ldots,\left(\frac{s_{n}}{s}\right) t_{n}\right)_{t}=$ $\left(\left(\frac{s_{0}}{s}, \ldots, \frac{s_{n}}{s}\right)\left(t_{0}, \ldots, t_{n}\right)\right)_{t}=\left(\left(\frac{s_{0}}{s}, \ldots, \frac{s_{n}}{s}\right)_{t}\left(t_{0}, \ldots, t_{n}\right)_{t}\right)_{t}=\left(t_{0}, \ldots, t_{n}\right)_{t}$ where the second equality follows from [1, Lemma 3.1]. Hence $\left(A_{f^{\prime}} D_{T}\right)_{t}=\left(A_{f^{\prime}}\right)_{t} D_{T}=D_{T}$ where the first equality follows from [1, Corollary 3.5]. It remains to show that $s^{\prime} D[X] \cap f^{\prime} D[X]=s^{\prime} f^{\prime} D[X]$ for all $s^{\prime} \in S$. It suffices to show that if $s \in S$ and $f \in D[X]$ with $\left(A_{f} D_{T}\right)_{t}=D_{T}$, then $s D[X] \cap f D[X]=s f D[X]$. Now since $S$ is an lcm splitting set, $D_{T}$ is a GCD domain. Since $f$ is primitive in $D_{T}[X]$, $s D_{T}[X] \cap f D_{T}[X]=s f D_{T}[X]$. Hence $s D[X] \cap f D[X]=\left(s D_{S}[X] \cap s D_{T}[X]\right) \cap$ $\left(f D_{S}[X] \cap f D_{T}[X]\right)=f D_{S}[X] \cap\left(s D_{T}[X] \cap f D_{T}[X]\right)=f D_{S}[X] \cap s f D_{T}[X]=$ sf $D_{S}[X] \cap s f D_{T}[X]=s f D[X]$. So $S$ is a splitting set in $D[X]$. Hence $S$ is an lcm splitting set of $D[X]$. Let $T^{\prime}$ be the $m$-complement of $S$ in $D[X]$. We claim that $T^{\prime}=\left\{0 \neq f \in D[X] \mid\left(A_{f} D_{T}\right)_{t}=D_{T}\right\}$. We have already shown the $\supseteq$ containment. Let $g \in T^{\prime}$. We have shown that $g=s g^{\prime}$ where $s \in S$ and $\left(A_{g^{\prime}} D_{T}\right)_{t}=D_{T}$. Since $g=1 g=s g^{\prime}$ where $1, s \in S$ and $g, g^{\prime} \in T^{\prime}, 1$ and $s$ are associates. Hence $s$ is a unit, so $\left(A_{g} D_{T}\right)_{t}=D_{T}$.

We next give another characterization of splitting sets.

Proposition 2.3. Let $S$ be a saturated multiplicatively closed subset of $D$ and let $T$ be the $m$-complement of $S$. Then $S$ is a splitting set if and only if (1) the set $S T$ is saturated and (2) every nonzero prime ideal $P$ that is disjoint with $S$ intersects $T$ nontrivially.

Proof. $(\Rightarrow)$ Let $S$ be a splitting set and suppose that $T$ is the $m$-complement of $S$. Then as $S T=D^{*}, S T$ is saturated. Next suppose that a nonzero prime ideal $P$ is disjoint with $S$ and let $d$ be a nonzero element of $P$. Then as $S$ is a splitting set, $d=s t$ where $s \in S$ and $t \in T$. Since $P \cap S=\varnothing, s \notin P$, so $t \in P$.

$(\Leftarrow)$ Suppose that $S$ is a saturated multiplicatively closed subset of $D$ and that (1) and (2) hold. We claim that $S T=D^{*}$. For if not, there is a nonzero $x \in D-S T$. Because $S T$ is saturated, a standard argument leads to the conclusion that there is a prime ideal $P$ containing $x$ such that $P \cap S T=\varnothing$. This implies that $P \cap S=\varnothing$. However, by (2) $P$ must intersect $T$ nontrivially which means that $P \cap S T \neq \varnothing$, a contradiction.

An element $x$ of $D^{*}$ is called primal if in $D, x \mid a b$ implies that $x=r s$ where $r \mid a$ and $s \mid b$. An element $p$ of $D$ is called completely primal if every factor of $p$ is primal. An integral domain with all nonzero elements primal is called pre-Schreier [13]. An integrally closed pre-Schreier domain was initially introduced as a Schreier 
ring by Cohn [6]. In general if $S$ and $T$ are saturated multiplicatively closed sets, it is not necessary that $S T$ should also be one. In fact, there is no relationship between $S$ and $T$ being saturated and $S T$ being saturated. Let $D$ be a Dedekind domain with class group $\mathrm{Cl}(D)=\mathbb{Z}_{2}$ and nonprincipal maximal ideals $M$ and $N$. Let $M^{2}=a D, N^{2}=b D$ and $M N=c D$. Then $S=\left\{u a^{n} \mid u \in U(D), n \geq 0\right\}$ and $T=\left\{u b^{n} \mid u \in U(D), n \geq 0\right\}$ are saturated multiplicatively closed subsets of $D$, but $S T$ is not saturated. For $c^{2} D=(M N)^{2}=M^{2} N^{2}=a b D$ gives $c^{2} \in S T$ but $c \notin S T$. Conversely, if $S T$ is saturated, then $S$ or $T$ may or may not be saturated, even in the domain $\mathbb{Z}$. Let $S=\{1\} \cup\left\{ \pm 2^{n}\right\}_{n \geq 1}$ and $T=\{ \pm 1\} \cup\left\{ \pm 2^{n}\right\}_{n>2}$, so $S T=\left\{ \pm 2^{n}\right\}_{n>0}$ is saturated while neither $S$ nor $T$ is saturated. If $S=\left\{2^{n}\right\}_{n>0}$ and $T=\left\{ \pm 3^{n}\right\}_{n>0}, T$ and $S T=\left\{ \pm 2^{n} \cdot 3^{m}\right\}_{n, m>0}$ are saturated, but $S$ is not saturated. Also, if $S=\left\{ \pm 2^{n}\right\}_{n \geq 0}$ and $T=\left\{ \pm 3^{n}\right\}_{n \geq 0}$, then $S, T$, and $S T$ are saturated. However, the following proposition gives a case where we can conclude that the product is saturated.

Proposition 2.4. Let $S$ be a saturated multiplicatively closed subset of $D$ consisting of completely primal elements and let $T$ be any saturated multiplicatively closed subset of D. Then ST is a saturated multiplicatively closed subset of D.

Proof. Clearly $S T$ is multiplicatively closed. Now let $y$ be a factor of an element $s t$ of $S T$. Then there exists $r \in D$ such that $r y=s t$. Since $s$ is completely primal and $s \mid r y, s=u v$ where $u \mid r$ and $v \mid y$. Let $y=y_{1} v$ and $r=r_{1} u$. Substituting in the original equation and cancelling out the common factors from both sides we conclude that $y_{1} \mid t$.

Corollary 2.5. Let $S$ be a saturated multiplicatively closed subset of $D$ consisting of completely primal elements. Then $S$ is a splitting set if and only if for each prime $P$ of $D$ that is disjoint with $S, P$ contains an element $x$ such that $x$ is v-coprime to $S$.

Proof. Let $T$ be the $m$-complement of $S$. As $S$ and $T$ are both saturated, so is $S T$ by Proposition 2.4 and now Proposition 2.3 applies.

Now obviously every prime element is completely primal and so are the so-called (lcm) extractors, i.e., elements $r$ of $D^{*}$ such that for each $x \in D, r D \cap x D$ is principal. There are a number of results in the literature that can be given somewhat different statements. Of these the following are worth mentioning. The reader may consult [1, Theorem 4.3] for Corollary 2.6 below. Note that the hypothesis of both corollaries gives that an element $x$ is $v$-coprime to $S \Longleftrightarrow x$ is coprime to $S$, i.e., $\operatorname{GCD}(x, s)=1$ for all $s \in S$.

Corollary 2.6. Let $S$ be a saturated multiplicatively closed subset of $D$ consisting of extractors. If $D_{S}$ is a GCD domain (respectively, PVMD) and every nonzero prime ideal of $D$ that is disjoint from $S$ contains an element coprime to $S$, then $D$ is a GCD domain (respectively, PVMD).

Corollary 2.7 (An alternative form of Nagata-like theorems). Let $S$ be a multiplicatively closed subset of D generated by primes. Suppose also that every nonzero prime ideal of $D$ that is disjoint from $S$ contains an element coprime to every element of $S$. If $D_{S}$ is a UFD (respectively, PVMD, GCD domain, atomic domain, etc.), then so is $D$. 
For the proof of this corollary the reader may note that the hypothesis ensures that $S$ is a splitting set. The rest has been amply established in the literature. The reader may consult [2, Theorem 3.1] for a quick reference/proof.

Corollary 2.7 provides a statement of Nagata's Theorem on UFD's that is worth mentioning because in this statement there is no reference to the ascending chain condition on principal ideals (ACCP) which is usually required for such results. Indeed, Corollary 2.7 also avoids any mention of the condition that $S$ be a splitting set as used in [2].

In an effort to complete the picture we study the effect $S$ being a splitting set has on the prime $t$-ideals of $D$. For this we take another look at 14. Immediately after the statement of [14, Theorem 1] appears the following statement: "As a consequence of the proof of this theorem we conclude that if $D^{(S)}\left[=D+X D_{S}[X]\right]$ is a GCD domain and if $P$ is a PF-prime of $D$ with $P \cap D \neq \varnothing$ then $P$ intersects $S$ in detail". (Recall that $P$ intersects $S$ in detail if for every nonzero prime ideal $Q \subseteq P, Q \cap S \neq \varnothing$.) In the more general situation we do not have the luxury of PF-primes, but we do have the advantage of hindsight. Hence we have the following proposition.

Proposition 2.8. Let $S$ be a multiplicatively closed subset of $D$ with the property that every nonzero prime $P$ disjoint with $S$ contains an element v-coprime to $S$. Then every prime $t$-ideal that intersects $S$ intersects it in detail. Consequently, if $S$ is a splitting set, then every prime $t$-ideal that intersects $S$, intersects $S$ in detail.

Proof. Suppose that $M$ is a prime $t$-ideal such that $M \cap S \neq \varnothing$. Let $s \in M \cap S$ and suppose that there is a nonzero prime ideal $Q$ contained in $M$ such that $Q \cap S=\varnothing$. By the hypothesis there is $d \in Q$ such that $d$ is $v$-coprime with $S$. But then $(s, d)_{v}=D$ and $s, d \in M$, contradicting the assumption that $M$ is a $t$-ideal.

That the converse of Proposition 2.8 is false was established in [14, Example 2.6]. However, the hypothesis of Proposition 2.8 may play an important role in deciding whether a multiplicatively closed set is a splitting set. We now proceed to show that even that is not enough to establish that the set in question is a splitting set. Before we do that, however, it seems pertinent to give the hypothesis of Proposition 2.8 a name. A multiplicatively closed set $S$ of an integral domain $D$ will be said to have property $*$ if for every nonzero prime $t$-ideal $P$ with $P \cap S=\varnothing, P$ contains an element $v$-coprime with $S$. It is routine to check that property $*$ and the hypothesis of Proposition 2.8 are equivalent. It is also routine to check that if $S$ has property*, then $S$ partitions $t$-max $(D)$ into two sets: $F=\{P \in t-\max (D) \mid P \cap S=\varnothing\}$ and $G=\{Q \in t-\max (D) \mid Q \cap S \neq \varnothing\}=\{Q \in t-\max (D) \mid Q$ intersects $S$ in detail $\}$ such that for all $P \in F$ and for all $Q \in G, P$ and $Q$ do not contain a common nonzero prime ideal. (Note that $F=\varnothing$ precisely when $S=D^{*}$ while $G=\varnothing$ precisely when $S=U(D)$.) Now let us establish that even $*$ is not enough to force $S$ to be a splitting set.

Example 2.9. Let $D$ be a Dedekind domain with torsion class group. Then for every nonzero prime ideal $P$ of $D$ there is a smallest natural number $n$ such that $P^{n}=(d)$. Next, as every power of $(d)$ is $P$-primary, we conclude that $S=\left\{u d^{m} \mid\right.$ $u \in U(D), m \geq 0\}$ is a saturated multiplicatively closed set. Clearly $S$ satisfies property $*$ because for every nonzero prime ideal $Q$ with $Q \cap S=\varnothing$, and for some $m \in \mathbb{N}, Q^{m}=(e)$ where $e \notin P$ and hence is $v$-coprime to $d$. Now let $n>1$. Then there is an atom $x \in P-\{0\}$ that is not $P$-primary. Obviously $x \notin S$ and being 
an atom, $x$ is incapable of being written as $x=s t$ where $s \in S$ and $t$ is $v$-coprime to $S$.

Now that we know that something less than a splitting set induces a partition of $t$-max $(D)$, we want to know what kind of partition of $t$-max $(D)$ is induced by a splitting set. The following theorem provides an answer.

Theorem 2.10. If $S \neq D^{*}, U(D)$ is a splitting set of $D$, then $S$ partitions $t$-max $(D)$ into $F=\{P \in t$-max $(D) \mid P \cap S=\varnothing\}$ and $G=\{Q \in t$-max $(D) \mid Q$ intersects $S$ in detail\} such that for $P \in F$ and $Q \in G$ if a nonzero element $x$ belongs to $P \cap Q$, then $x=x_{1} y_{1}$ where $x_{1} \in P-X$ for every $X \in G$ and $y_{1} \in Q-Y$ for every $Y \in F$. Conversely, let $S \neq D^{*}, U(D)$ be a saturated multiplicatively closed set and let $F=\{P \in t$-max $(D) \mid P \cap S=\varnothing\}$ and $G=\{Q \in t$-max $(D) \mid Q$ intersects $S$ in detail $\}$. Suppose $t-\max (D)=F \cup G$ and if $0 \neq x \in P \cap Q$ where $P \in F$ and $Q \in G$, then $x=x_{1} y_{1}$ where $x_{1} \in P-X$ for all $X \in G$ and $y_{1} \in Q-Y$ for all $Y \in F$. Then $S$ is a splitting set and $\{F, G\}$ is a partition of $t$-max $(D)$.

Proof. Indeed, if $S$ is a splitting set, then $S$ satisfies $*$ and hence $t$-max $(D)$ is partitioned into $F$ and $G$ as described in the statement of the theorem. Now as $S$ is a splitting set, given $P \in F, Q \in G$ and given $0 \neq x \in P \cap Q, x=x_{1} y_{1}$ where $y_{1} \in S$ and $x_{1}$ is $v$-coprime to every member of $S$. Because two $v$-coprime elements cannot share a prime $t$-ideal, $x_{1}$ does not belong to any member of $G$. Hence $x_{1} \in P-X$ for all $X \in G$. This also implies that $y_{1} \in Q$. That $y_{1} \in Q-Y$ for all $Y \in F$ follows from the fact that $y_{1} \in S$.

Conversely, let $P \in F$. As $P \cap S=\varnothing, S \subseteq D-P$. So $S \subseteq \bigcap_{P \in F}(D-P)=$ $D-\bigcup_{P \in F} P$ and as $S$ is a saturated multiplicatively closed set, $S=D-\bigcup_{P \in F} P$. (For if not, there is a nonzero $x \in\left(D-\bigcup_{P \in F} P\right)-S$ and as $S$ is saturated, a minimal prime ideal $q$ of $(x)$ that is disjoint with $S$. Now $q$ being a $t$-ideal must be contained in one of the maximal $t$-ideals. Clearly as $q$ is not contained in any members of $F, q$ must be contained in some member of $G$. But every member of $G$ intersects $S$ in detail, a contradiction.)

Next let $T=D-\bigcup_{Q \in G} Q$ and consider $S T$. We claim that $S T=D^{*}$. For if not, let $x \in D^{*}-S T$. Then as $x \notin S T, x \notin S$ and $x \notin T$ and hence there is at least one $P \in F$ and at least one $Q \in G$ such that $x \in P \cap Q$. But then by the given property, $x=x_{1} y_{1}$ where $x_{1} \in P-X$ for all $X \in G$ and $y_{1} \in Q-Y$ for all $Y \in F$. Since $x_{1} \in P-X$ implies that $x_{1} \in D-X$ we conclude that $x_{1} \in T=D-\bigcup_{Q \in G} Q$ and similarly $y_{1} \in S=D-\bigcup_{P \in F} P$. But this implies that $x=x_{1} y_{1} \in S T$ and thus $D^{*}=S T$. So every nonzero nonunit of $D$ can be written as $u v$ where $u \in S$ and $v \in T$. Now to complete the proof we must show that $v$ is $v$-coprime to every element of $S$. For this let $s \in S$ and consider $(s, v)$. Noting that $s$ is not in any member of $F$ and $v$ is not in any member of $G$ and that $F \cup G=t$-max $(D)$ we conclude that $s$ and $v$ share no maximal $t$-ideal of $D$. Hence $(s, v)_{v}=(s, v)_{t}=D$. Thus we have established that $S$ is a splitting set. That $\{F, G\}$ is a partition follows from the very definition of $F$ and $G$.

Corollary 2.11. Let $S \neq D^{*}, U(D)$ be a splitting set of $D$. Then

$$
S=D-\bigcup\{P \in t-\max (D) \mid P \cap S=\varnothing\}
$$

with m-complement

$$
T=D-\bigcup\{Q \in t-\max (D) \mid Q \cap S \neq \varnothing\} .
$$


Proof. This follows from the proof of Theorem 2.10,

Theorem 2.12. Suppose that every nonzero nonunit of $D$ belongs to only a finite number of maximal $t$-ideals and suppose that $t$-max $(D)$ is partitioned into two sets $F$ and $G$ such that for every $(P, Q) \in F \times G$, and for every nonzero $x \in P \cap Q$, $x=p q$ where $p \in P-Q$ and $q \in Q-P$. Then $S=D-\left(\bigcup_{P \in F} P\right)$ is a splitting set with $m$-complement $T=D-\left(\bigcup_{Q \in G} Q\right)$. Conversely, if $S \neq D^{*}, U(D)$ is a splitting set with $m$-complement $T$, then $S$ induces a partition of $t$-max $(D)$ into two disjoint sets $F=\{P \in t$-max $(D) \mid P \cap S=\varnothing\}$ and $G=\{Q \in t$-max $(D) \mid Q$ intersects $S$ in detail $\}$ such that for every $(P, Q) \in F \times G$, and for every nonzero $x \in P \cap Q$, $x=p q$ where $p \in P-Q$ and $q \in Q-P$.

Proof. Let $F$ and $G$ be as given and let $x$ be a nonzero nonunit of $D$. Then $x$ belongs to only a finite set of maximal $t$-ideals, say $x \in P_{1}, P_{2}, \ldots, P_{r}, Q_{1}, Q_{2}, \ldots, Q_{s}$, where the $P_{j}$ 's $\in F$ and $Q_{i}$ 's $\in G$. We show that $x \in S T$ by induction on $r+s$. If $r+s=1$, the result is clear. If $r=0$ or $s=0$, clearly $x \in S T$. So suppose $r \geq 1$ and $s \geq 1$. Then by the condition, $x=x_{1} y_{1}$ where $x_{1} \in P_{1}-Q_{1}$ and $y_{1} \in Q_{1}-P_{1}$. Now $x_{1}$ and $y_{1}$ are each contained in at most $r+s-1$ maximal $t$-ideals. So by induction $x_{1}, y_{1} \in S T$. Hence $x=x_{1} y_{1} \in S T$. The converse is obvious.

Recall that an integral domain $D$ is weakly Krull if $D=\bigcap_{P \in X^{(1)}} D_{P}$ where $X^{(1)}=X^{(1)}(D)$ is the set of height-one primes of $D$ and the intersection is locally finite. Thus a Cohen-Macaulay integral domain is an atomic weakly Krull domain.

Corollary 2.13. Let $D$ be an atomic weakly Krull domain and let $\{F, G\}$ be a partition of $X^{(1)}(D)$. Then $S=D-\left(\bigcup_{P \in F} P\right)$ is a splitting set if and only if no member of $F$ shares an atom with any member of $G$.

In [4, Theorem] we showed that for an integral domain $D$ the following conditions are equivalent: (1) each saturated multiplicatively closed subset of $D$ is a splitting set, (2) for each prime ideal $P$ of $D, S=D-P$ is a splitting set, and (3) $D$ is weakly Krull and $\mathrm{Cl}_{t}(D)=0$. Here $\mathrm{Cl}_{t}(D)$ denotes the $t$-class group of $D$ - the group of $t$-invertible $t$-ideals of $D$ modulo the subgroup of principal fractional ideals. Since according to Corollary 2.11 a splitting set $S \neq D^{*}$ of $D$ must be the complement of a union of maximal $t$-ideals, it is of interest to characterize the integral domains for which each such multiplicatively closed set is a splitting set. Several conditions equivalent to (3) of Theorem 2.14 are given in [3, Corollary 4.4].

Theorem 2.14. For an integral domain $D$ the following conditions are equivalent.

(1) For each nonempty collection $F$ of maximal t-ideals of $D, S=D-\bigcup_{P \in F} P$ is a splitting set.

(2) For each maximal t-ideal $P$ of $D, S=D-P$ is a splitting set.

(3) $D=\bigcap_{P \in t-\max (D)} D_{P}$ has finite character, for distinct $P, Q \in t$-max $(D)$, $P \cap Q$ contains no nonzero prime ideal, and $\mathrm{Cl}_{t}(D)=0$.

Proof. (10) $\Rightarrow$ (2) Clear. (2) $\Rightarrow$ (3) Let $P \in t$-max $(D)$. Since $S=D-P$ is a splitting set, by Theorem $1.2 x D_{P} \cap D$ is principal for each $x \in D$. The implication now follows from [3, Corollary 4.4]. (3) $\Rightarrow$ (11) Suppose that $D$ satisfies the hypotheses of (3). Let $F$ be as in (11) and let $G=t$-max $(D)-F$. We may assume $G \neq \varnothing$ for $G=\varnothing$ gives $S=U(D)$. We apply Theorem [2.12, Let $0 \neq x \in P \cap Q$ where $(P, Q) \in F \times G$. By [3, Corollary 4.4] we can write $x=x_{1} \cdots x_{n}$ where each $x_{i}$ is contained in a unique maximal t-ideal $P_{i}$ (for by [3, Lemma 4.2] if $x_{i}$ is $P_{i}$-pure 
where $P_{i} \in t$-max $(D)$, then $P_{i}$ is the unique maximal $t$-ideal containing $\left.x_{i}\right)$. Let $p=\prod\left\{x_{i} \notin Q\right\}$ and $q=\prod\left\{x_{i} \mid x_{i} \notin P\right\}$. Then $x=p q$ where $p \in P-Q$ and $q \in Q-P$.

\section{ACKNOWLEDGEMENT}

We would like to thank the referee for several observations that simplified the proofs of Theorems 2.1 and 2.2.

\section{REFERENCES}

1. D.D. Anderson, D.F. Anderson, and M. Zafrullah, Splitting the t-class group, J. Pure Appl. Algebra 74 (1991), 17-37. MR 93d:13023

2. D.D. Anderson, D.F. Anderson, and M. Zafrullah, Factorization in integral domains, II, J. Algebra 152 (1992), 78-93. MR 94c:13019

3. D.D. Anderson, J.L. Mott, and M. Zafrullah, Finite character representations for integral domains, Boll. Un. Mat. Ital. B (7) 6 (1992), 613-630. MR 93k:13001

4. D.D. Anderson and M. Zafrullah, Weakly factorial domains and groups of divisibility, Proc. Amer. Math. Soc. 109 (1990), 907-913. MR 90k:13015

5. D.F. Anderson, J. Park, G.-I. Kim, and H.-J. Oh, Splitting multiplicative sets and elasticity, Comm. Algebra 26 (1998), 1257-1276. MR 99c:13002

6. P.M. Cohn, Bezout rings and their subrings, Proc. Cambridge Philos. Soc. 64 (1968), 251-264. MR 36:5117

7. S. Gabelli and M. Roitman, On Nagata's theorem for the class group, J. Pure Appl. Algebra 66 (1990), 31-42. MR 91i:13015

8. R. Gilmer and T. Parker, Divisibility properties in semigroup rings, Michigan Math. J. 21 (1974), 65-86. MR 49:7381

9. J.L. Mott, The group of divisibility of Rees rings, Math. Japon. 20 (1975), 85-87. MR 52:10718

10. J.L. Mott and M. Schexnayder, Exact sequences of semi-value groups, J. Reine Angew. Math. $\mathbf{2 8 3 / 2 8 4}$ (1976), 388-401. MR 53:8050

11. M. Nagata, A remark on the unique factorization theorem, J. Math. Soc. Japan 9 (1957), 143-145. MR 18:869a

12. M. Roitman, On Mori domains and commutative rings with $\mathrm{CC}^{\perp} \mathrm{I}$, J. Pure Appl. Algebra 56 (1989), 247-268. MR 90c:13019

13. M. Zafrullah, On a property of pre-Schreier domains, Comm. Algebra 15 (1987), 1895-1920. MR 88g:13018

14. M. Zafrullah, The $D+X D_{S}[X]$ construction from $G C D$-domains, J. Pure Appl. Algebra 50 (1988), 93-107. MR 89k:13017

Department of Mathematics, The University of Iowa, Iowa City, Iowa 52242

E-mail address: dan-anderson@uiowa.edu

Department of Mathematics, SCEn 301, The University of Arkansas, Fayetteville, ARKANSAS 72701

E-mail address: kamla@compuserve.com

Current address: Department of Mathematics, Campus Box 8085, Idaho State University, Pocatello, Idaho 83209

E-mail address: mzafrullah@usa.net 